\title{
Periodic electrochemical oscillations at a solid-solid electrode
}

\author{
Stefan Majoni, Jürgen Janek* \\ Institut für Physikalische Chemie und Elektrochemie und Sonderforschungsbereich 173 der Universität Hannover, Callinstr. 3-3A, \\ 30167 Hannover, Germany
}

\begin{abstract}
During the anodic dissolution of a silver electrode in the transference cell $\mathrm{Ag}|\boldsymbol{\alpha}-\mathrm{AgI}| \mathrm{Ag}$, periodic changes of the anodic overvoltage have been observed under galvanostatic conditions. The conditions for the occurrence of these electrochemical oscillations are described. We compare the observed behaviour with similar phenomena at passivating metal electrodes in liquid electrolytes.
\end{abstract}

Keywords: Interface; Electrochemical oscillations; Solid electrolyte; $\alpha$-AgI

\section{Introduction}

Solid-solid electrodes play an important role in the construction and the behaviour of solid state galvanic cells, and the development of solid electrolytes was always connected to the investigation of related electrode properties [1]. The kinetics of electrodes, made of a metal $\mathrm{A}$, in contact with cation conducting solid electrolytes AX, in particular, have been investigated intensively for many years (e.g. [2-4]), aiming for a description of the electrode kinetics in terms of well established models in the framework of liquid state electrochemistry.

We present an example of a very unusual electrode behaviour in the solid state, namely the occurrence of periodic electrochemical oscillations at the interface between a cation conducting solid electrolyte and the parent metal anode. As an excellent example

\footnotetext{
* Corresponding author.

Email: janek@mbox.pci.uni-hannover.de
}

for the existence of dissipative structures, periodic electrochemical oscillations are well known for certain metal electrodes in liquid electrolytes since almost hundred years $[5,6]$, but to our knowledge have not yet been reported for the solid state.

\section{Experimental}

The standard cell arrangement is depicted schematically in Fig. 1. It consists of a cylindrical silver iodide pellet (prepared by precipitation from $\mathrm{AgNO}_{3}$ and $\mathrm{KI}$ solutions), two silver electrodes (99.999\% Ag, Demetron, Germany), and a small potential probe (silver wire) which was embedded in the solid electrolyte near the anode.

The whole cell was placed in a sample holder (glass) which allowed the variable application of mechanical pressure (along the cell) by the use of metal springs. Constant current was supplied to the cell by means of a galvanostat (Jaissle 10000 T-B), 


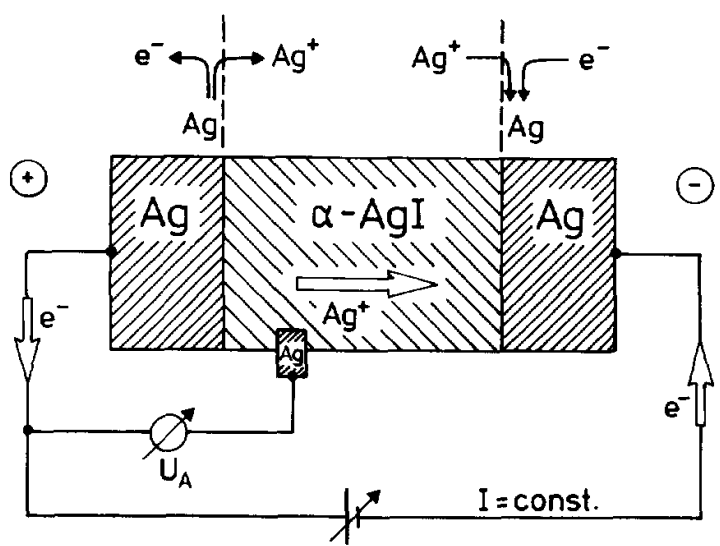

Fig. 1. Schematic cell arrangement for the electrodissolution experiment.

and the experimental data were collected by the use of a personal computer equipped with an A/D converter.

Neither the surface treatment nor the crystallinity of the anode was critical for the occurrence of electrochemical oscillations. In most cases, we used silver electrodes which were firstly mechanically and finally electrochemically polished.

Experiments were performed in the temperature range $150-400^{\circ} \mathrm{C}$, and the mechanical pressure was varied between 1 and 7 bar. The geometrical electrode area was typically $0.2 \mathrm{~cm}^{2}$, and the applied current densities were in the order of $1 \mathrm{~mA} / \mathrm{cm}^{2}$. Under galvanostatic conditions, the voltage $U_{\mathrm{A}}(t)$ between the anode and the small probe in the electrolyte was the main experimental quantity. For further experimental details see Ref. [7].

\section{Results}

Depending on the experimental parameters, the time development of the voltage $U_{\mathrm{A}}(t)$ showed different behaviour. Most critical for the occurrence of periodic voltage oscillations are the current density and the applied mechanical pressure. At low applied pressures, $U_{\mathrm{A}}(t)$ generally increased appreciably after a certain transient time and finally reached a plateau. Superposed on this plateau we observed irregular oscillations with high frequencies and rather small amplitudes (cf. Fig. 2).

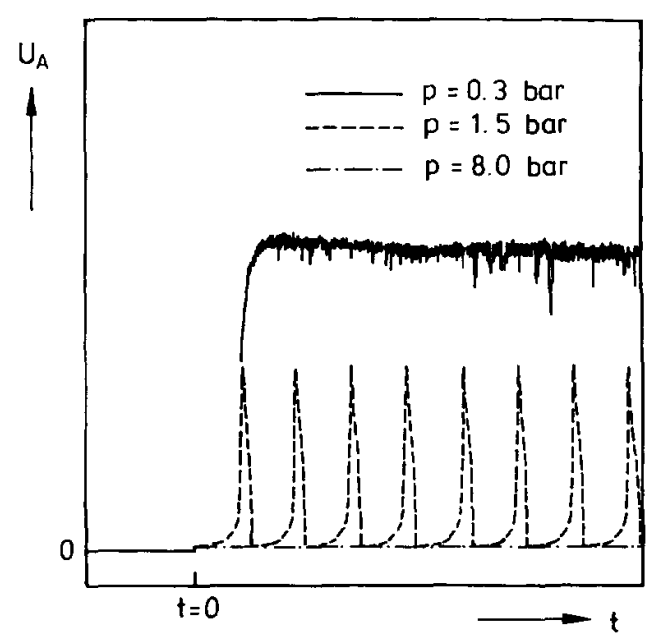

Fig. 2. Qualitative behaviour of the voltage $U_{A}(t)$ between the silver anode and the silver potential probe as a function of time for three different applied mechanical pressures, $T=260^{\circ} \mathrm{C}$.

At pressures higher than $7 \mathrm{bar}$, the voltage $U_{\mathrm{A}}(t)$ generally remained stable at its original low value. In the pressure range between these two cases (approximately 1-7 bar) we were able to obtain a steady oscillatory behaviour which is depicted in Fig. 2. In addition, another example of the observed voltage oscillations is depicted in Fig. 3.

The galvanostatic voltage oscillations were qualitatively reproducible, but differed in detail from one experiment to another. Thus, it was very difficult to make systematic investigations of the variation of the oscillatory modes as a function of the experimental parameters. Nevertheless, at least some clear qualitative conclusions can be drawn. The frequencies of the oscillations increased with increasing current density and usually took values in the order of some $10^{1}-10^{2} \mathrm{~s}^{-1}$. With increasing temperature, the amplitudes decreased. Besides, the amplitudes of the oscillations varied considerably from one experiment to another, but generally remained smaller than the thermodynamic decomposition voltage of $\mathrm{AgI}$, which equals approximately $0.64 \mathrm{~V}$ at $260^{\circ} \mathrm{C}$ and a iodine activity of 1 .

Due to additional experiments with varying conditions (purity of silver, crystallinity of silver, oxygen content of silver, surface preparation, etc. [3]) we conclude that the voltage oscillations are caused by the intrinsic transport properties of the $\mathrm{Ag} \mid \alpha-\mathrm{AgI}$ 


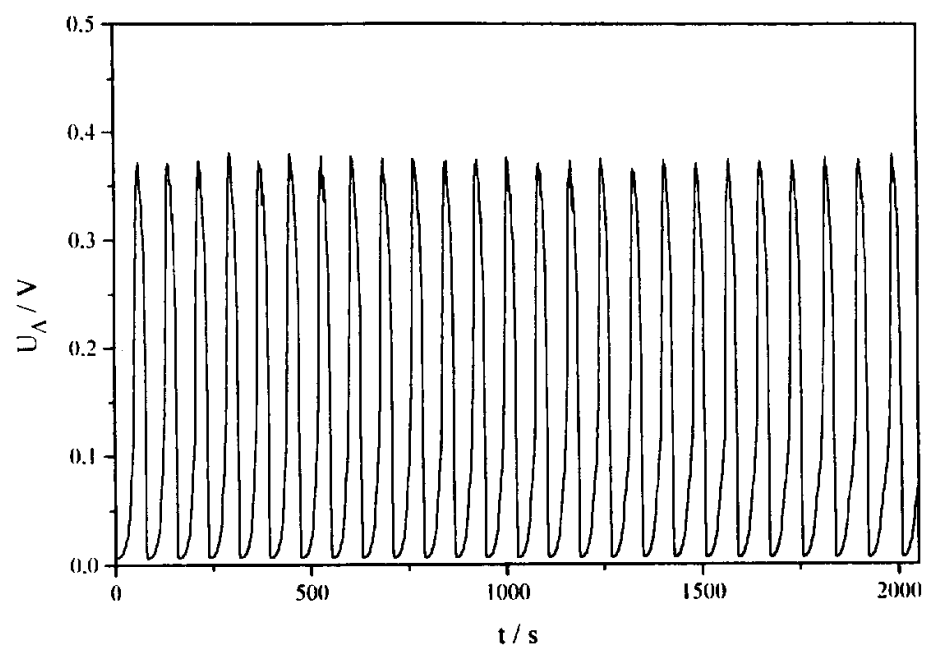

Fig. 3. Representative galvanostatic measurement of $U_{\mathrm{A}}(t) ; j=1.56 \mathrm{~mA} / \mathrm{cm}^{2}, T=260^{\circ} \mathrm{C}$, applied mechanical pressure: 2 bar.

interface, i.e., the oscillations have to be explained by the respective interface kinetics.

\section{Discussion}

The basic ionic transfer process at the anodic interface can be described by the ion exchange equilibrium

$$
\begin{aligned}
\operatorname{Ag}_{A g}(A g)= & {\left[A g_{A g}^{\times}(A g I)-V_{A g}^{\prime}(A g I)\right]+e^{\prime}(A g) } \\
& +V_{A g}(A g)
\end{aligned}
$$

which is formulated using the Kröger-Vink notation. The structure elements $A g_{\mathrm{Ag}}(\mathrm{Ag})$ and $\mathrm{V}_{\mathrm{Ag}}(\mathrm{Ag})$ represent a metal atom and a lattice vacancy in the metal, $\mathrm{e}^{\prime}(\mathrm{Ag})$ denotes an electron in the metal, and $\mathrm{Ag}_{\mathrm{Ag}} \times$ (AgI) and $\mathrm{V}_{\mathrm{Ag}}^{\prime}(\mathrm{AgI})$ represent a metal ion and a cation vacancy in the compound AgI, respectively. Essentially, the structure element combination $\left[\mathrm{Ag}_{\mathrm{Ag}}^{\times}(\mathrm{AgI})-\mathrm{V}_{\mathrm{Ag}}^{\prime}(\mathrm{AgI})\right]$ means a silver cation in the solid electrolyte. Each metal atom that is transferred across the interface during the anodic process leaves a vacant site in the metal at the interface and occupies a vacant cation site in the electrolyte. A constant dissolution rate, i.e. a constant ionic current across the solid electrolyte, in principle leads to a constant rate of vacancy production at the interface. Depending on the annihilation and transport pro- cesses of the vacancies, the interface may degrade due to the accumulation of vacancies. At a temperature of $800^{\circ} \mathrm{C}$ the anodic process may even lead to the injection of vacancies into the metal, as it was shown for the case of $\mathrm{Ag} \mid \mathrm{Ag}-\boldsymbol{\beta}-\mathrm{Al}_{2} \mathrm{O}_{3}$ by Fischbach [8]. At lower temperatures, the mobility of vacancies in the metal bulk and at the interface is generally lower, leading to the accumulation of vacancies. Thus, in the galvanostatic case, the dissolution of silver is, in principle, a self inhibiting process. It produces vacancies at the interface which reduce the active interface area and, therefore, increase the local current densities at the remaining contact areas. We believe that this self inhibition is at least phenomenologically equivalent to the passivation of metals in liquid electrolytes by the creation of isolating surface layers [6]. With this hypothesis in mind, several aspects of the oscillating interface system have to be investigated more closely to prove our assumption.

Firstly, the proof of a passivating "vacancy accumulation" (pores) has to be given experimentally. The periodic creation and removal of passivating surface layers on metal electrodes in liquid electrolytes can be investigated favourably by the use of ellipsometry [9] or probably by in situ scanning tunnelling microscopy. Unfortunately, no comparable techniques are available for the study of interfaces between solids. Ex situ scanning electron microscope 
pictures of the interface show a fine porous structure of the metal anode surface after an experiment. These support our idea, but yield no kinetic information. Thus, trying to measure the rate of advancement of the receding anodic interface, we performed a high precision dilatometric in situ study of the anodic dissolution process [10]. The results give clear evidence of the successive creation and removal of pore volume at the interface.

Secondly, the mechanism of relaxation of the interface, which again leads to the decrease of the voltage $U_{\mathrm{A}}(t)$ after the creation of a high-resistance structure, has to be explained. This step is far more difficult to understand and to interpret unequivocally, than the degradation process itself, which is a necessary consequence of the transfer process (cf. Eq. (1)). Several mechanisms are imaginable, some being more obvious than others. Perhaps the simplest model is based on the assumption of a mechanical breakdown of the degraded interface structure when a critical state is reached. We believe that such breakdown of a porous structure would not result in the observed highly regular oscillatory voltage signal, but rather should lead to an irregular voltage signal. Also, other simple models are not able to explain all aspects of the observed phenomenon [11].

In the present state, we believe that a combination of at least two transport mechanisms, i.e. conventional interface transfer and surface diffusion, have to be taken into account for the interpretation of the observed phenomenon.

A final comment has to be given on the strong correlation of the local microscopic transfer processes. The measured voltage $U_{\mathrm{A}}(t)$ is an average value for the whole electrode area, which equals approximately $0.2 \mathrm{~cm}^{2}$. The typical length of the structures of the degraded interface is only some microns. Thus, a strong and long-range interaction seems to correlate the local processes. The identification of this interaction is also part of our future work.

\section{Conclusions}

It is shown that a constant anodic ionic current across the $\mathrm{Ag} \mid \alpha$-AgI interface may result in periodic oscillations of the voltage across the interface. To our knowledge, this is the first observation of a regular electrochemical oscillation in the solid state.

We conclude that the oscillations are the direct consequence of a periodically changing interface structure. A strong interaction of the local microscopic transfer processes must exist to explain the correlated behaviour of the macroscopic interface as a whole. Both the mechanism of this correlation and the relaxation mechanism of the degraded interface during the oscillations are not understood completely in the present state.

Thus, further experimental and theoretical investigations are in progress, to clarify the origin of the observed oscillatory interface kinetics.

\section{Acknowledgments}

The authors gratefully acknowledge the support of Prof. H. Schmalzried. Financial support from the Fonds der Chemischen Industrie (Germany) is also gratefully acknowledged.

\section{References}

[1] C. Deportes et al., Electrochimie des Solides (Presses Universitaires de Grenoble, Grenoble 1994) p. 4

[2] D.O. Raleigh, in: Solid State Batteries Devices, Proc. NATO ASI Ser., W. van Gool, ed. (North-Holland, Amsterdam, 1972) p. 477.

[3] R.D. Armstrong, T. Dickinson and P.M. Willis, J. Electroanal. Chem. 57 (1974) 231.

[4] E.A. Ukshe and N.G. Bukun, Elektrokhimiya 16 (1980) 313; 26 (1990) 1373.

[5] U.F. Franck, Angew. Chem. 90 (1978) 1.

[6] S.K. Scott, Chemical Chaos (Clarendon Press, Oxford, 1993) p. 11.

[7] J. Janek and S. Majoni, Ber. Bunsenges. Phys. Chem. 99 (1995) 14.

[8] H. Fischbach, Z. Metallk. 71 (1980) 115.

[9] L.T. Tsitsopoulos, T.T. Tsotsis and I.A. Webster, Surf. Sci. 191 (1987) 225.

[10] S. Majoni and J. Janek, submitted to Ber. Bunsenges. Phys. Chem.

[11] S. Majoni, PhD Thesis, University of Hannover, 1995. 